\title{
ToxA Is Present in the U.S. Bipolaris sorokiniana Population and Is a Significant Virulence Factor on Wheat Harboring Tsn1
}

T. L. Friesen ${ }^{\dagger}$ and D. J. Holmes, Cereal Crops Research Unit, Red River Valley Agricultural Research Center, USDA-ARS, Fargo, ND; R. L. Bowden, Hard Winter Wheat Genetics Research Unit, Center for Grain and Animal Health Research, USDA-ARS, Manhattan, KS; and J. D. Faris, Cereal Crops Research Unit, Red River Valley Agricultural Research Center, USDA-ARS, Fargo, ND

\begin{abstract}
ToxA, a necrotrophic effector originally identified from the tan spot fungus Pyrenophora tritici-repentis in 1987, was subsequently identified from Parastagonospora nodorum in 2006. More recently, the ToxA gene was identified in the spot blotch fungus Bipolaris sorokiniana in Australia. Here we show that the ToxA gene is also present in the $B$. sorokiniana population in the winter wheat region of southcentral Texas. Leaves from 'Duster' wheat showing strong necrotic lesions were collected in Castroville, TX. Fifteen single-spore isolates were collected from separate lesions, and 13 of them harbored the BsToxA gene and secreted ToxA in culture based on sensitivity of BG261, the differential line containing the dominant ToxA sensitivity gene, Tsn1. Four isolates harboring BsToxA and one deficient in BsToxA were used to infiltrate two wheat lines harboring $T s n 1$ as well as their corresponding $t s n 1$ mutant lines. Culture filtrates of the isolate lacking

BsToxA did not induce necrosis on any of the lines. Culture filtrates of the four BsToxA-containing isolates induced necrosis on the wild type (Tsn1) lines but not on the corresponding tsn 1 mutant lines. Sensitivity to these culture filtrates also mapped to the previously identified location for $T s n 1$ in the winter wheat mapping population Arina $\times$ Forno. Inoculation of one of these ToxA-producing isolates on the same population showed that the Tsn1 locus accounted for $24.4 \%$ of the disease variation. All 13 isolates harbored the same BsToxA nucleotide sequence, which was identical to one of the two haplotypes previously identified in Australia. Sensitivity to ToxA is prevalent in popular hard winter wheat cultivars in the central and southcentral winter wheat regions of the United States, showing the potential of a selective advantage for $B$. sorokiniana isolates that harbor the ToxA gene.
\end{abstract}

Helminthosporium leaf blight of wheat is a foliar disease complex made up of the necrotrophic fungal pathogens Pyrenophora triticirepentis and Bipolaris sorokiniana, causal agents of tan spot and spot blotch on wheat, respectively. In a 2-year field study located in Rampur, Nepal, Duveiller et al. (2005) used six cultivars to show that grain yield losses ranged from 17 to $55 \%$ depending on the cultivar used, with complete crop loss possible in warm high-rainfall years, making Helminthosporium leaf blight the main limiting factor for wheat production in this and surrounding regions. B. sorokiniana typically thrives under warm humid conditions and is a major constraint to wheat production in warm humid climates such as South Asia, where B. sorokiniana is more damaging than other foliar pathogens such as P. tritici-repentis (Duveiller and Dubin 2000). B. sorokiniana, however, has been of minimal importance in countries such as the United States, Canada, and Australia, where P. tritici-repentis has been more prevalent (Duveiller and Dubin 2000).

The ToxA protein was first discovered by Tomás and Bockus (1987) using culture filtrates of $P$. tritici-repentis infiltrated on wheat lines ranging from resistant to highly susceptible. Culture filtrates induced necrosis in a differential manner that correlated strongly with disease susceptibility. Several groups worked toward the purification of this necrosis-inducing protein, which was ultimately named Ptr ToxA (Ciuffetti et al. 1998). Ptr ToxA is a 13.2-kDa proteinaceous necrotrophic effector, the first protein necrotrophic effector to be discovered (Tuori et al. 1995). Using the protein sequence, two groups eventually cloned the ToxA gene (Ballance et al. 1996; Ciuffetti et al. 1997 ) and showed that it was a single copy gene in the $P$. triticirepentis genome. Friesen et al. (2006) later identified a nearly identical ToxA gene in Parastagonospora nodorum (causal agent of septoria nodorum blotch) and showed that ToxA had likely been horizontally

${ }^{\dagger}$ Corresponding author: T. L. Friesen; E-mail: timothy.friesen@ ars.usda.gov Accepted for publication 16 May 2018.

This article is in the public domain and not copyrightable. It may be freely reprinted with customary crediting of the source. The American Phytopathological Society, 2018. transferred from $P$. nodorum to $P$. tritici-repentis. More recently, McDonald et al. (2012) showed that a third fungal species, Parastagonospora avenaria tritici, contained the ToxA gene and suggested that it was likely obtained through interspecific hybridization.

Host sensitivity to Ptr ToxA was mapped to wheat chromosome arm 5BL with the sensitivity gene being named Tsnl (Faris et al. 1996). This gene was a significant factor in both tan spot and septoria nodorum blotch when the infecting pathogens harbored the ToxA gene (Friesen et al. 2006; Lamari and Bernier 1989). Faris et al (2010) cloned Tsnl and found that it had similarities to classical resistance-like genes, including nucleotide binding, leucine-rich repeat, and protein kinase domains. This was significant because it demonstrated that a resistance-like gene conferred susceptibility rather than resistance to $P$. tritici-repentis and $P$. nodorum isolates expressing ToxA (Friesen et al. 2006).

McDonald et al. (2017) recently discovered a functional ToxA gene in several Australian isolates of B. sorokiniana. Two nucleotide haplotypes were identified differing by only a single nonsynonymous nucleotide substitution, with one of these haplotypes being identical to the most prevalent $P$. tritici-repentis haplotype (Ciuffetti et al. 1997). This work showed that transfer of virulence genes between species infecting the same host may be more common than previously thought.

In 2016, we collected leaves from winter wheat cultivar 'Duster' from winter wheat research plots in Castroville, TX, that were covered with necrotic lesions that contained almost exclusively $B$. sorokiniana. Here we show that ToxA was present in the B. sorokiniana population of the southcentral winter wheat region of Texas and that, similar to $P$. tritici-repentis and $P$. nodorum, $B$. sorokiniana isolates harboring ToxA are effectively using the ToxA-Tsn1 interaction to increase the level of disease on wheat. Given the high prevalence of Tsn1 in popular hard winter wheat cultivars, this discovery has serious implications for wheat production in the United States, especially in the central and southcentral winter wheat regions of the United States.

\section{Materials and Methods}

Pathogen identification. Leaves of the winter wheat cultivar Duster with distinct necrotic lesions were collected from cooperative research plots operated by Texas A\&M University in Castroville, 
TX, on 20 April 2016 to identify what pathogen was causing these unique spots. Approximately 10 dry leaves were surface sterilized as described in Liu et al. (2012). Surface-sterilized leaves were placed on water agar under a 12-h photoperiod at room temperature to induce sporulation. Conidia forming out of the necrotic lesions were collected under a stereoscope using a dissecting needle that was flame sterilized and cooled in water agar. Several single spores from each of 15 distinctly separate lesions were transferred to V8 potato dextrose agar (PDA) and allowed to grow. A single colony was randomly chosen from each of the 15 lesions for a second round of single-spore isolation to ensure purity. The fungus was identified based on conidia and conidiophore morphology.

Amplification of ToxA from Texas B. sorokiniana isolates. Fifteen $B$. sorokiniana isolates collected from leaves as described above were used to investigate the presence of ToxA. DNA of each isolate was extracted using the BioSprint 15 DNA Plant Kit (Qiagen). The ToxA sequence was polymerase chain reaction (PCR) amplified using ToxA primers 226 bp upstream and $161 \mathrm{bp}$ downstream of the start and stop codons, respectively, as described in Friesen et al. (2006). The internal transcribed spacer (ITS) sequence was amplified as a positive control, using primers ITS 1 and ITS 4 (White et al. 1990). A second attempt at ToxA amplification was made for any isolate that did not yield ToxA amplicons in the first experiment.

Production and infiltrations of $\operatorname{SnToxA}$ and $B$. sorokiniana culture filtrates. The $15 \mathrm{~B}$. sorokiniana isolates were used to prepare culture filtrates as previously described (Friesen and Faris 2012). Briefly, spores were produced by transferring dried mycelial disks to V8-PDA medium and grown in 24-h light until spores appeared in abundance, typically 5 to 7 days. Approximately 200,000 spores were harvested and inoculated into $60 \mathrm{ml}$ of Fries medium (Liu et al. 2006) and grown with shaking at $27^{\circ} \mathrm{C}$ for $72 \mathrm{~h}$. Cultures were transferred to a dark growth chamber at $21^{\circ} \mathrm{C}$ and incubated for an additional week. Culture filtrates were harvested by filtering each flask's contents through a Fisher Scientific Qualitative P5 filter followed by a Millipore Durapore $0.45-\mu \mathrm{m}$ HV filter. Purified SnToxA was prepared and used as described in Friesen and Faris (2012). Undiluted filtrates as described above were infiltrated using a 1-ml needleless syringe into various wheat lines harboring Tsn1 or tsn1 to verify the presence of BsToxA in B. sorokiniana culture filtrates. Purified SnToxA was used as a positive control as well as to identify the presence of $T s n 1$ in uncharacterized wheat lines.

Validation of the presence of ToxA in culture filtrates using Tsn1 and tsn1 mutant lines. Wheat lines harboring Tsn1 including Bobwhite and Langdon along with their corresponding tsn1 mutant lines (Faris et al. 2010) were also used to validate the presence of BsToxA in culture filtrates of the Texas B. sorokiniana isolates. Infiltrations were done as described above.

Production of spores for inoculation. B. sorokiniana spores were harvested after 5 days of growth on V8-PDA as described in Faris and Friesen (2005) and diluted to 3,000 spores/ml with one drop of Tween 20 per $50 \mathrm{ml}$ of inoculum to reduce spore clumping. The spore suspension was inoculated until runoff, at which time plants were placed in chambers at $100 \%$ humidity for $24 \mathrm{~h}$ followed by 6 days in a $21^{\circ} \mathrm{C}$ growth chamber under a 12 -h photoperiod. Plants were evaluated on a 1 to 5 scale similar to the tan spot of wheat scale developed by Lamari and Bernier (1989).

Evaluation of plant materials for ToxA sensitivity and disease severity. A collection of winter (W) and spring (S) type wheat lines from diverse breeding backgrounds was used to identify the presence of Tsnl (ToxA sensitivity). These lines included Iba (W), Jamestown (W), Duster (W), BR34 (S), Ruby Lee (W), ND495 (S), Jerry (W), Salamouni (S), and W7984 (synthetic hexaploid) as well as mapping population parental lines Arina (W), and Forno (W) (Table 1). Additionally, this collection of lines was used in inoculation experiments to evaluate their reaction to isolate TexD16Bs1, which was positive for the ToxA gene. The Tsnl differential line BG261 (Friesen et al. 2006) was used as a ToxA-sensitive control.

Twenty-two and 20 of the most popular hard red winter wheat cultivars planted in Texas and Oklahoma, respectively, were chosen based on the Texas Wheat Varieties 2017 and the 2017 Oklahoma Wheat Variety Reports accessed at https://www.nass.usda.gov. Lines were evaluated for ToxA sensitivity and therefore the prevalence of Tsn1. Lines were infiltrated with purified SnToxA at the two- to three-leaf stage as described above.

Mapping sensitivity to culture filtrates and susceptibility to spore inoculations of $\boldsymbol{B}$. sorokiniana. A 120 -line $\mathrm{F}_{5}$ recombinant inbred mapping population developed from a cross of the Swiss winter wheat cultivars Arina and Forno (Paillard et al. 2003) was provided by Beat Keller, University of Zurich, Switzerland, and used to map sensitivity to $B$. sorokiniana culture filtrates and susceptibility to spore inoculations. Culture filtrates of TexD16Bs1 were infiltrated across the Arina $\times$ Forno mapping population, which segregates for ToxA sensitivity versus insensitivity (Tsn1/tsn1) (Abeysekara et al. 2009). Additionally, purified SnToxA was infiltrated side by side as a control. Culture filtrate and SnToxA sensitivity were mapped in the Arina $\times$ Forno population as described by Abeysekara et al. (2009).

The Arina $\times$ Forno population and parental lines were also grown and subjected to spore inoculation as described by Abeysekara et al. (2009). The BsToxA-producing isolate TexD16Bs1 was used to inoculate the Arina $\times$ Forno population as described above. A total of three replications were completed for all inoculations, and two replications were completed for all infiltrations. The mean lesion types from the three replications of spore inoculations for the recombinant inbred lines were used to identify significant marker-trait associations using multiple interval mapping in the quantitative trait locus (QTL) analysis program QGene version 4.3 (Joehanes and Nelson 2008). The marker dataset for the Arina $\times$ Forno population consisted of 460 markers and was the same as that used by Abeysekara et al. (2009). The critical logarithm of the odds threshold of 3.8 for an experiment-wise significance at the 0.01 level of probability was determined by conducting 1,000 permutations.

\section{Results}

Identification of $B$. sorokiniana from wheat lesions. Duster wheat grown in Castroville, TX, contained an abundance of 3 to

Table 1. Diverse set of wheat lines evaluated for ToxA sensitivity using purified SnToxA; these lines were also evaluated for susceptibility to a Texas Bipolaris sorokiniana isolate harboring the ToxA gene (see Figure 2)

\begin{tabular}{llll}
\hline Wheat line & \multicolumn{1}{c}{ Wheat type } & \multicolumn{1}{c}{ Origin } & ToxA sensitive \\
\hline Iba & Hard red winter & Oklahoma State University & + \\
Jamestown & Soft red winter & Virginia Tech & - \\
Duster & Hard red winter & Oklahoma State University & + \\
BR34 & Spring & Brazil & - \\
Ruby Lee & Hard red winter & Oklahoma State University & + \\
ND495 & Hard red spring & North Dakota State University & + \\
Jerry & Hard red winter & North Dakota State University & + \\
Salamouni & Soft spring & Landrace & - \\
W7984 & Synthetic hexaploid & CIMMYT & - \\
Arina & Winter & Swiss & - \\
Forno & Winter & Swiss & + \\
\hline
\end{tabular}


$5 \mathrm{~mm}$ necrotic lesions (Fig. 1A). Spores emerging from surfacesterilized leaves were almost exclusively $B$. sorokiniana and were clearly identified as such based on their typical abundant sporulation (Fig. 1B) and the heavily brown-pigmented oblong, septate conidia (Fig. 1C). Conidia isolated from lesions were reinoculated to show that Duster is highly susceptible to these isolates (Fig. 2). Fifteen $B$. sorokiniana isolates were single-spored to be used in downstream experiments and were named based on their collection location Texas (Tex), cultivar Duster (D), year 2016 (16), and isolates being identified as B. sorokiniana (Bs) (i.e., TexD16Bs1 to TexD16Bs15).

Identification of BsToxA and production of BsToxA in Texas B. sorokiniana isolates. Based on the necrotic lesion phenotype found on Duster, which harbors Tsn1, and the recent discovery of ToxA in B. sorokiniana in Australia (McDonald et al. 2017), we used PCR to attempt to amplify ToxA from the $15 \mathrm{~B}$. sorokiniana isolates. ToxA was amplified from 13 of the 15 isolates (Fig. 3). Culture filtrates of the 13 isolates harboring ToxA identified by PCR induced
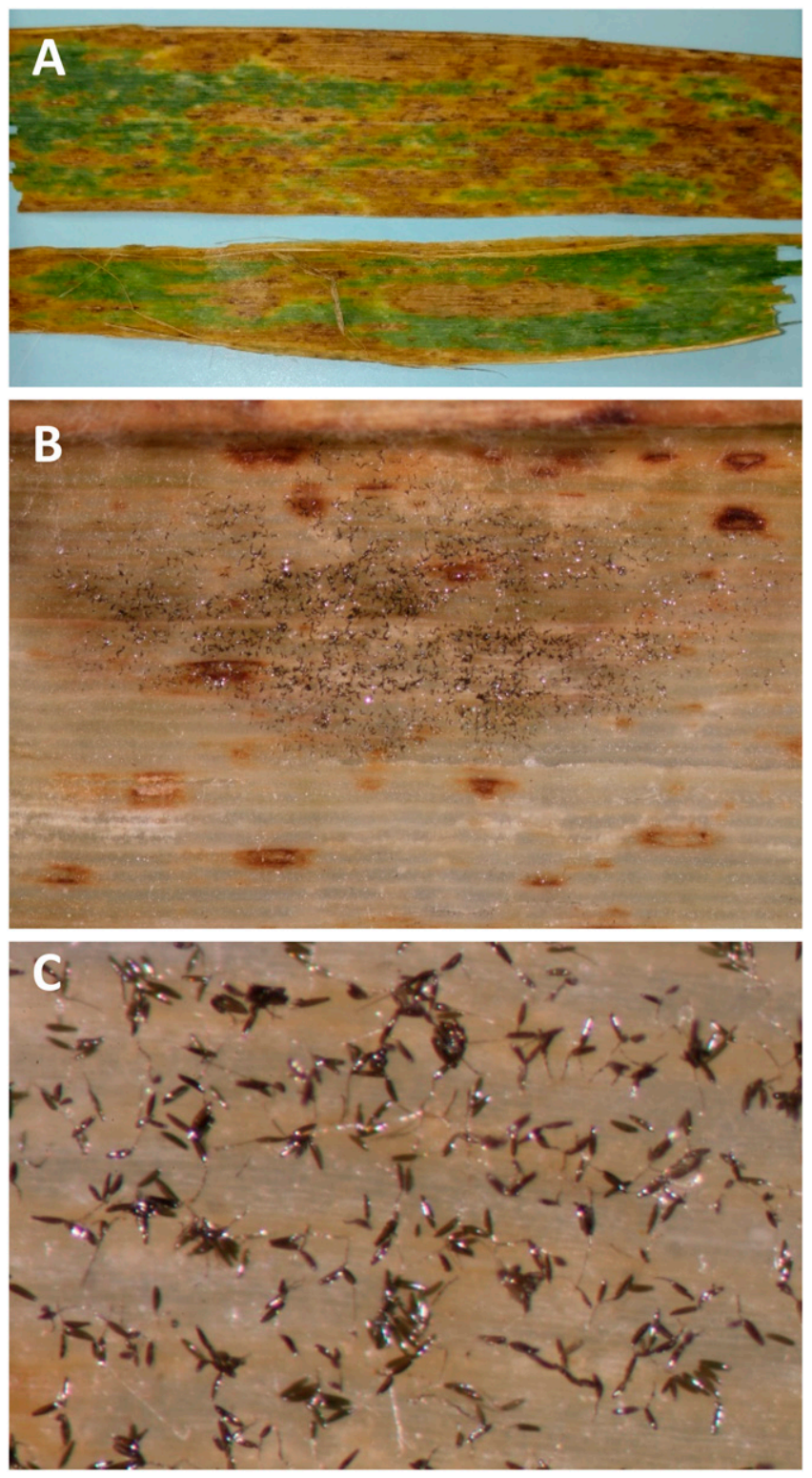

Fig. 1. Isolation of Bipolaris sorokiniana from leaf samples of Duster wheat collected in Castroville, TX, in 2016. A, Field leaves of the hard red winter wheat line Duster heavily affected by necrotic lesions induced by $B$. sorokiniana. B, A lesion after being surface sterilized and incubated on water agar for 5 days showing the production of darkly pigmented spores typical of the spot blotch fungus $B$. sorokiniana. C, Close-up view of the $B$. sorokiniana conidiophores and conidia protruding from the infected leaf surface. necrosis when infiltrated on both Duster wheat and the Tsn1 differential line BG261; however, the two isolates deficient in ToxA showed no necrosis on either line.

B. sorokiniana inoculation on a selection of wheat lines. Several wheat lines from different classes and breeding programs were evaluated for ToxA sensitivity (Tsn1) and susceptibility to $B$. sorokiniana (Fig. 2). Five of these lines had Tsn1 and four did not. In this limited set, lines harboring $T s n 1$ were visibly more susceptible than lines lacking Tsn1. This result led us to further evaluate the Arina $\times$ Forno

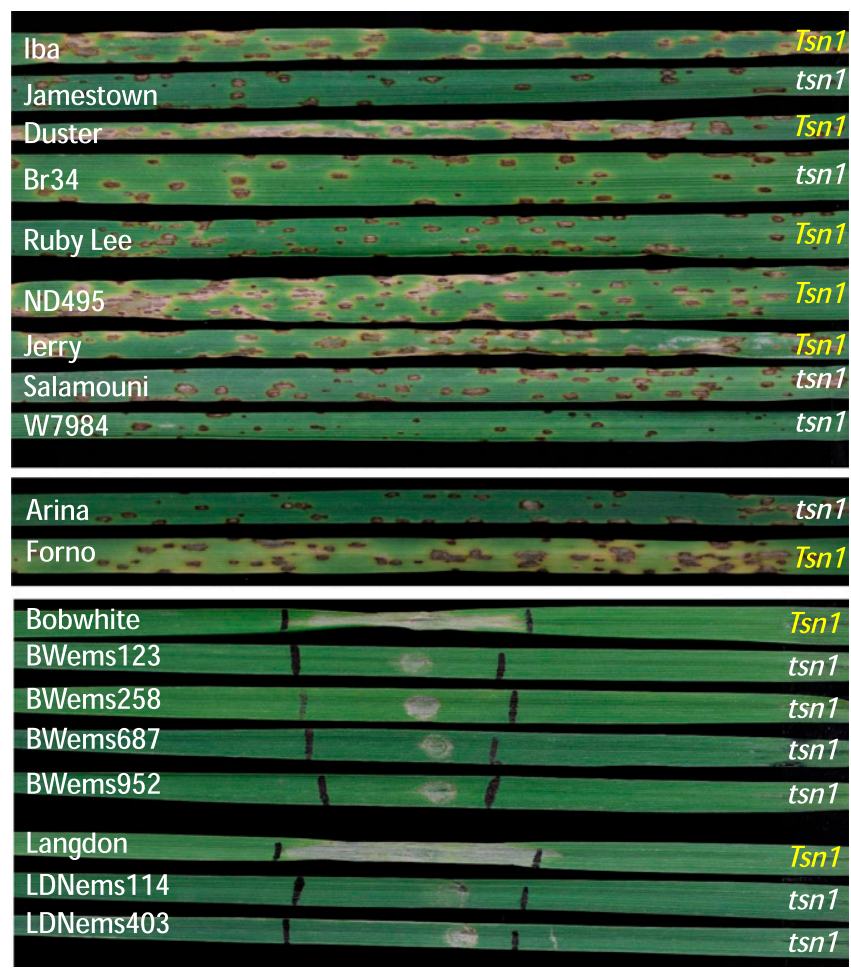

Fig. 2. Seedling disease reactions of wheat lines with and without Tsn1 infiltrated or inoculated with culture filtrates or spores of isolate TexD16Bs1, respectively. Top panel, spore inoculations on various winter and spring wheat lines using Bipolaris sorokiniana isolate TexD16Bs1. Middle panel, winter wheat lines Arina and Forno inoculated with isolate TexD16Bs1. Bottom panel, infiltration of Bobwhite and Langdon, both of which harbor Tsn1, along with four tsn1 mutants of Bobwhite and two tsn1 mutants of Langdon using culture filtrates of isolate TexD16Bs1.

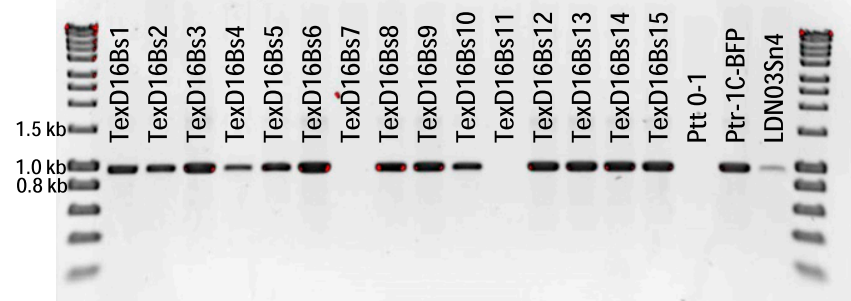

Fig. 3. Polymerase chain reaction amplification of ToxA from 15 Bipolaris sorokiniana isolates (TexD16Bs1 to TexD16Bs15) collected from necrotic lesions on Duster wheat in Castroville, TX. Controls include Pyrenophora teres f. teres (Ptt0-1) lacking ToxA, and P. tritici-repentis (Ptr-1C-BFP) and Parastagonospora nodorum (LDN03Sn4), both of which harbor ToxA. 
mapping population to see if Tsnl was indeed contributing to spot blotch susceptibility in wheat.

Infiltration of B. sorokiniana culture filtrates on $T s n 1$ and $t s n 1$ mutant wheat lines. Five isolates were randomly chosen for additional culture filtrate production and analysis. Culture filtrates of five of the isolates, four of which harbored the ToxA gene and one of which did not, were infiltrated on the Tsn1 lines Bobwhite and Langdon as well as the previously characterized Bobwhite and Langdon tsn 1 ethyl methanesulfonate (ems)-induced mutant lines (Faris et al.
2010). Culture filtrates of TexD16Bs7, which was deficient in ToxA, did not induce necrosis on any of the lines; however, culture filtrates of the isolates harboring the BsToxA gene (TexD16Bs1, TexD16Bs4, TexD16Bs6, and TexD16Bs9) induced necrosis on Bobwhite and Langdon but not on the tsn1 mutant lines (LDNems114, LDNems403, BWems123, BWems258, BWems687, and BWems952) (Fig. 2), showing that BsToxA was interacting with $T s n 1$ alone. This serves as additional evidence that the BsToxA protein was interacting with Tsn1, resulting in necrosis.
TexBsToxA
AusBsToxA1
AusBsToxA2
Pt-1C-BFP_ToxA
Sn4_ToxA

TexBstoxA

AusBsToxA1

AusBsToxA2

Pt-1C-BFP TOXA

Sn4_ToxA

TexBsToxA

AusBstoxA1

AusBstoxA2

Pt-1C-BFP_TOXA

Sn4_ToxA

TexBsToxA

AusBstoxA1

AusBstoxA2

Pt-1C-BFP TOXA

Sn4_ToxA

TexBs ToxA

AusBsToxA1

AusBsToxA2

Pt-1C-BFP_TOXA

Sn4_ToxA

TexBsToxA

AusBsToxA1

AusBsToxA2

Pt-1C-BFP ToxA

Sn4_ToxA

TexBstoxA

AusBstoxA1

AusBstoxA2

Pt-1C-BFP_TOXA

Sn4_ToxA

TexBstoxA

AusBsToxA1

AusBsToxA2

Pt-1C-BFP_TOXA

Sn4_ToxA

TexBstoxA

AusBstoxA1

AusBsToxA2

Pt-1C-BFP_TOXA

Sn4_ToxA

TexBstoxA

AusBstoxA1

AusBsToxA2

Pt-1C-BFP_TOXA

Sn4_ToxA atgcgttctatcctcgtacttctttcagcgecgetgctgtgcttgctgccccaacgect

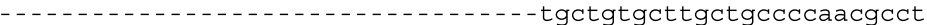
- - - - - - - - - - - - - - - agcgecgetgctgtgcttgctgccccaacgcct atgcgttctatcctcgtacttctttcagcgccgctgctgtgcttgctgccccaacgcet atgcgttctatcctcgtacttctttcagcgccgctgctgtgcttgctgccccaacgcet $\star * * * * * * * * * * * * * * * * * * * * * * * *$

gaagccgatcccggttacgaaatcgttaaacttttcgaagccgccaactcttctgaactc gaagccgatcccggttacgaaatcgttaaactttccgaagccgccaactcttctgaactc gaagccgatcccggttacgaaatcgttaaactttcgaagccgccaactcttctgaactc gaagccgatcccggttacgaaatcgttaaacttttcgaagccgccaactcttctgaactc gaagccgatcccggttacgaaatcgttaaactttcgaagccgccaactcttctgaactc

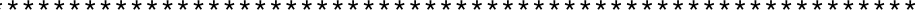

gacgcgcgcggactctctctcgactggaccctcaaaccgcgcggactcctacaggagcgg gacgcgcgcggactctctctcgactggaccctcaaaccgcgcggactcctacaggagcgg gacgcgcgcggactctctctcgactggaccctcaaaccgcgcggactcctacaggagcgg gacgcgcgcggactctctctcgactggaccctcaaaccgcgcggactcctacaggagcgg gacgcgcgcggactctctctcgactggaccctcaaaccgcgcggactcctacaggagcgg

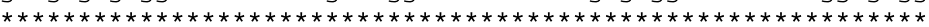

cagggaagctgcatgtcaatcacaatcaaccctagtcgtccgtctgtcaacaacatcggc cagggaagctgcatgtcaatcacaatcaaccctagtcgtccgtctgtcaacaacatcggc cagggaagctgcatgtcaatcacaatcaaccctagtcgtccgtctgtcaacaacatcggc cagggaagctgcatgtcaatcacaatcaaccctagtcgtccgtctgtcaacaacatcggc cagggaagctgcatgtcaatcacaatcaaccctagtcgtccgtctgtcaacaacatcggc

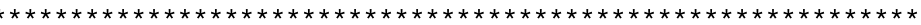

caagtcgacattgacagtgttatactcggacgacctggtgctataggctcgtgggaactg caagtcgacattgacagtgttatactcggacgacctggtgctataggctcgtgggaactg caagtcgacattgacagtgttatactcggacgacctggtgctataggctcgtgggaactg caagtcgacattgacagtgttatactcggacgacctggtgctataggctcgtgggaactg caagtcgacattgacagtgttatactcggacgacctggtgctataggctcgtgggatctg

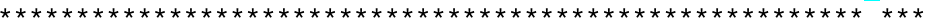

aacaactttattaccattggattgaaccgcgtaaacgccgatacagtgcgagtcaacatc aacaactt tattaccattggattgaaccgcgtaaacgccgatacagtgcgagtcaacatc a acaactttattaccattggattgaaccgcgtaaacgccgatacagtgcgagtcaacatc a aca actt tattaccattggattgaaccgcgtaaacgccgatacagtgcgagtcaacatc aacaact tat taccattggattgaaccgcgtaaacgccalatacagtgcgagtcaacatc

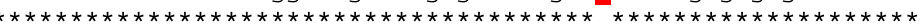

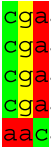

aacaccggcaggactaatcgcctcattattactcaatgggaataagtctccccacca acaccggcaggactaatcgcctcattattactcaltgggaataagtctccccacca aacaccggcaggactaatcgcctcattattactcaatgggaataagtctccccacca aacaccggcaggactaatcgcctcattattactcaatgggaataagtctccccacca aacaccggcaggactaatcgcctcattattactcaatgggaataagtctccccacca

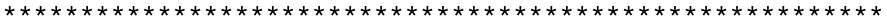

tttattagattacttattactaacttatactactaatactctcactcggggggacgttt ttttattagattacttattactaacttatactactaatactgtcactcggggggacgttt ttttattagattacttattactaacttatactactaatactctcactcggggggacgttt ttttattagattacttattactaacttatactactaatactgtcactcggggggacgttt ttt tattagattacttattactaacttatactactaatactctcactcggggggacgttt

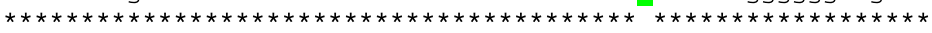

atgagcttttggtgattacgcttaattcaaggtagaggaagctttgccttaatatac atgagcttttggtgattacgcttaattcaaggtagaggaagctttgcctaatatac atgagctttttggtgattacgctttaattcaaggtagaggaagcttttgccttaatatac atgagcttttggtgattacgcttaattcaaggtagaggaagctttgccttaatatac atgagcttttggtgattacgctttaattcaaggtagaggaagcttttgccttaatatac

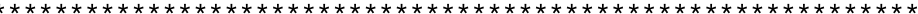

gatctgatacaggtcgtgaaattggagaatgcagctagaaaattag gatctg--.-- - - - - - - - - - - - - - - - - - - - - - -

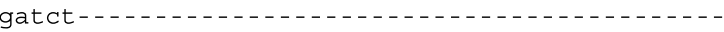
gatctgatacaggtcgtgaaattggagaatgcagctagaaattag gatctgattcaggtcgtgaaaattggagaatgcagctagaaaattag

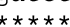

Fig. 4. Nucleotide sequences of BsToxA from Texas (TexBsToxA) and Australia (AusBsToxA1 and AusBsToxA2) aligned to Parastagonospora nodorum Sn4 (Sn4_ToxA) and Pyrenophora tritici-repentis (Pt-1C-BFP_ToxA) ToxA using Clustal Omega (Sievers et al. 2011). Variation between sequences is highlighted. 


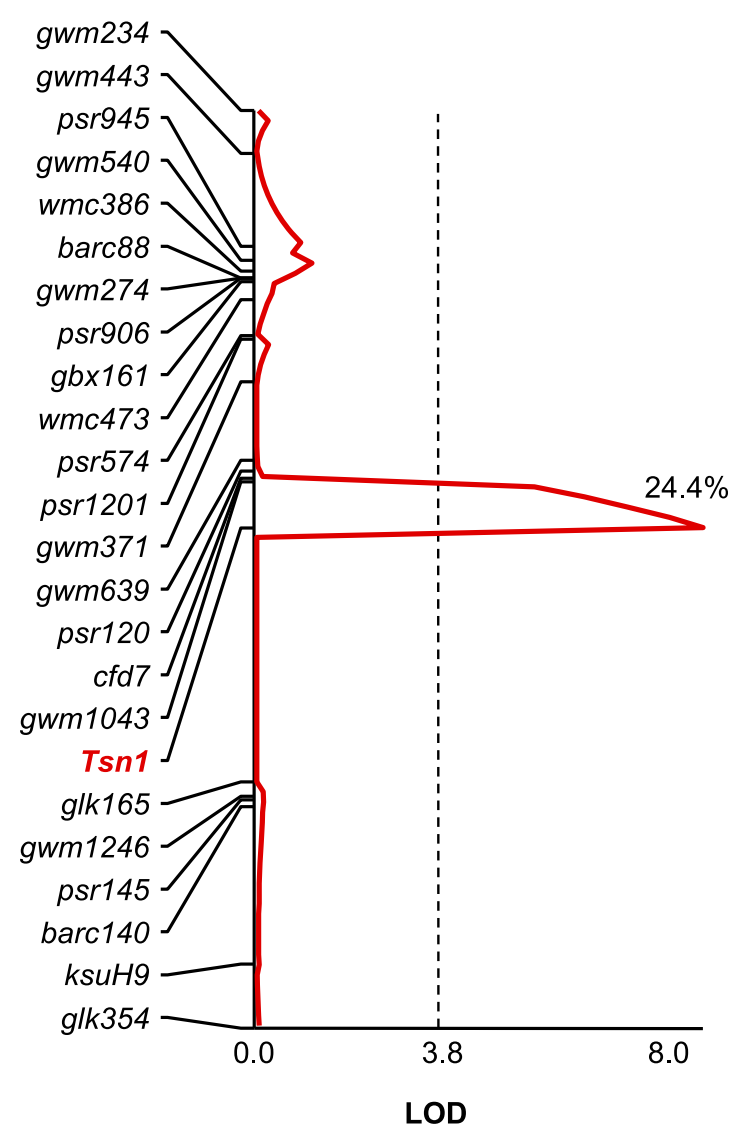

Fig. 5. Spot blotch disease association with Tsn1 (ToxA sensitivity) on chromosome 5B in the Arina $\times$ Forno recombinant inbred population using multiple interval mapping analysis. The logarithm of the odds (LOD) significance threshold of 3.8 is shown by a dashed line.
ToxA gene sequencing in the Texas $B$. sorokiniana isolates. ToxA was amplified and sequenced from the 13 isolates harboring the BsToxA gene. A single haplotype was identified across all 13 Texas isolates (Fig. 4), with this sequence differing by a single nucleotide from the most common $P$. tritici-repentis sequence (Ciuffetti et al. 1997) and being identical to one of the two BsToxA haplotypes identified in Australia (McDonald et al. 2017).

Mapping of sensitivity to BsToxA and susceptibility to $B$. sorokiniana using the Arina $\times$ Forno recombinant inbred line population. Purified SnToxA and culture filtrates of TexD16Bs1 were infiltrated side by side across the Arina $\times$ Forno mapping population. Sensitivity to purified SnToxA cosegregated perfectly with sensitivity to culture filtrates of TexD16Bs1 (Fig. 5). Both reactions mapped to wheat chromosome arm 5BL precisely where $T s n 1$ had previously been mapped (Abeysekara et al. 2009).

Multiple interval mapping of the mean lesion types from spore inoculations indicated that only one genomic region in the Arina $\times$ Forno population was significantly associated with disease resistance versus susceptibility, and that region was defined by the $T s n 1$ locus on 5BL (Fig. 5). Tsn1 defined the peak of the regression curve, and it had a logarithm of the odds of 8.0 and explained $24.4 \%$ of the disease variation.

ToxA sensitivity in popular Texas and Oklahoma winter wheats. Twenty-two of the 35 most popular hard red winter wheat lines in 2017 reported for Texas and 20 of the 35 most popular hard red winter wheat lines in 2017 reported for Oklahoma were infiltrated with purified SnToxA to identify the prevalence of $T s n 1$ in hard red winter wheat (Table 2). Due to overlap between the two sets, 26 lines were evaluated, with 20 of the 26 being sensitive to ToxA (Table 1). Additionally, the top four lines for acreage in Texas (TAM111, TAM112, TAM113, and Gallagher) and the top five lines for acreage in Oklahoma were all sensitive to ToxA (Table 1), showing the prevalence of Tsn1 in the hard red winter wheat cultivars of the south central United States.

\section{Discussion}

In this paper, we report the identification of a functional ToxA gene in a Texas population of $B$. sorokiniana. The ToxA gene had a high

Table 2. Reaction to ToxA infiltration of popular winter wheat cultivars planted in 2017 in Texas and Oklahoma

\begin{tabular}{|c|c|c|c|c|}
\hline TX rank order ${ }^{\mathbf{a}}$ & OK rank order ${ }^{\mathbf{a}}$ & Cultivar name & Breeding program origin & ToxA reaction $^{b}$ \\
\hline 1 & 17 & TAM111 & Texas A\&M & + \\
\hline 2 & 15 & TAM112 & Texas A\&M & + \\
\hline 3 & 33 & TAM113 & Texas A\&M & + \\
\hline 4 & 1 & Gallagher & Oklahoma State University & + \\
\hline 5 & 14 & WB Cedar & WestBred & - \\
\hline 6 & & Greer & Syngenta-Agripro & - \\
\hline 9 & & TAM401 & Texas A\&M & + \\
\hline 10 & & TAM105 & Texas A\&M & + \\
\hline 12 & 3 & Endurance & Oklahoma State University & + \\
\hline 13 & 34 & TAM204 & Texas A\&M & - \\
\hline 15 & 18 & Jackpot & Syngenta-Agripro & + \\
\hline 18 & 2 & Duster & Oklahoma State University & + \\
\hline 19 & 7 & Jagger & Kansas State University & + \\
\hline 20 & 19 & WB Grainfield & WestBred & + \\
\hline 21 & & TAM110 & Texas A\&M & + \\
\hline 22 & & TAM304 & Texas A\&M & - \\
\hline 23 & 9 & Winterhawk & Kansas State University & + \\
\hline 26 & 11 & WB 4458 & WestBred & - \\
\hline 27 & 4 & Iba & Oklahoma State University & + \\
\hline 29 & 8 & Fuller & Kansas State University & + \\
\hline 31 & & Hatcher & Colorado State University & + \\
\hline \multirow[t]{5}{*}{33} & 23 & Garrison & Oklahoma State University & + \\
\hline & 5 & Ruby Lee & Oklahoma State University & + \\
\hline & 13 & Everest & Kansas State University & - \\
\hline & 21 & Scout 66 & University of Nebraska & + \\
\hline & 22 & Overley & Kansas State University & + \\
\hline
\end{tabular}

\footnotetext{
a Indicates the rank order of popularity based on percentage of reported acres planted in Texas (https://www.nass.usda.gov/Statistics_by_State/Texas/index.php, accessed 10/24/2017) or Oklahoma (https://www.nass.usda.gov/Statistics_by_State/Oklahoma/Publications/Oklahoma_Wheat_Varieties/index.php, accessed $10 / 24 / 2017)$.

${ }^{\mathrm{b}}$ Reaction to infiltration of purified SnToxA. $+=$ sensitive, and $-=$ insensitive.
} 
degree of similarity to the ToxA gene identified in $P$. tritici-repentis (Ballance et al. 1996; Ciuffetti et al. 1997), P. nodorum (Friesen et al. 2006), and P. avenaria tritici (McDonald et al. 2012). Our results corroborate a recent report that the ToxA gene exists in Australian populations of B. sorokiniana (McDonald et al. 2017). The high degree of similarity of ToxA sequences among the four species can best be explained by horizontal gene transfer (Friesen et al. 2006) or interspecific hybridization (McDonald et al. 2017), and it is remarkable that this phenomenon has occurred at least three times in the Pleosporales. The exact match of the ToxA sequence of our isolates and the sequence of one haplotype described by McDonald et al. (2017) suggests that relatively recent global migration of these strains has occurred.

Spot blotch on wheat has been a major problem in warm, humid regions of the world; however, little is known about how the pathogen is infecting the host. Kumar et al. (2009) used a highly aggressive B. sorokiniana isolate collected in India to evaluate the genetics of spot blotch field resistance in a recombinant inbred line population developed from a cross of the resistant Chinese line Yangmai 6 and the Indian cultivar Sonalika. Sonalika was a popular cultivar in the 1970s and 1980s but was highly susceptible to spot blotch. A major-effect QTL on chromosome 5B, the same chromosome that harbors Tsn1, was identified. This QTL was isolated in a second population using a progeny recombinant inbred line backcrossed to the susceptible Sonalika (Kumar et al. 2015). Fine mapping in this population showed that this QTL mapped to the long arm of chromosome $5 \mathrm{BL}$ and was named $S b 2$. Interestingly, markers in the $S b 2$ region placed it in the same region as the ToxA sensitivity gene Tsn1 (Faris et al. 2010), indicating the possibility that the $S b 2$ susceptibility allele and Tsn1 are the same gene. We evaluated three accessions of Sonalika from the national small grains collection (PI 478282, PI 337371, and CItr 15392) for ToxA sensitivity and found that all three accessions were sensitive to purified ToxA (data not shown), showing that Sonalika does indeed carry $T s n 1$. This is significant because it shows that $B$. sorokiniana isolates in the regions where spot blotch has major economic impact may already harbor ToxA. If this is the case, it would be advisable to eliminate Tsn1 from all wheat lines planted in areas where spot blotch is a major problem.

Historically, this disease has not been of major concern in the United States or Canada. However, numerous distinct or coalescing necrotic lesions were identified on the popular hard red winter wheat cultivar Duster at a wheat breeding nursery in Castroville, TX. Leaves were collected, surface-sterilized, and incubated to identify the causal pathogen. Lesions contained almost exclusively $B$. sorokiniana, and the majority of the single-spore isolates collected harbored the BsToxA gene. The presence of the BsToxA gene in $B$. sorokiniana in the southern hard red winter wheat region is significant, especially because the majority of the most popular hard red winter wheat cultivars planted in Texas and Oklahoma harbor Tsn1 and are therefore potentially more vulnerable to all three of the wheat pathogens that employ ToxA as a virulence factor.

Several studies have shown $T s n 1$ to be a significant susceptibility factor for tan spot (Faris et al. 2013, review) and septoria nodorum blotch (Friesen et al. 2006; Liu et al. 2006; Virdi et al. 2016), but it has also been shown recently that the importance of this susceptibility factor is background dependent (Chu et al. 2008; Faris and Friesen 2005; Faris et al. 2012; Virdi et al. 2016). Here we show that Tsn1 is a significant susceptibility factor for spot blotch at the seedling stage in the winter wheat population Arina $\times$ Forno. Although the significance of the ToxA-Tsn1 interaction shown here in the Arina $\times$ Forno population was performed at the seedling stage, it is likely that this interaction is also significant at the adult plant stage because the distinct necrotic lesions from which the $B$. sorokiniana containing BsToxA was isolated were observed at the adult plant stage. Additional work will need to be done to investigate whether BsToxA is an effective virulence factor in other backgrounds including spring wheat and durum wheat and at other stages of growth.

It is thought that $P$. tritici-repentis, $P$. nodorum, and $B$. sorokiniana are differentially effective under different environmental conditions. Because $T s n l$ has never been shown to contribute positively to wheat, it would be advisable to select against Tsn1 to avoid the impact of this virulence factor present in three major wheat pathogens.

It is also important that these lesions were identified on Duster wheat, which harbors Tsn1 (ToxA sensitive) and is a popular cultivar in both Oklahoma and Texas. Here we also evaluated 26 of the most popular wheat lines planted in Texas and Oklahoma and found that 20 of these lines harbor Tsn1. Additionally, 12 of the top 25 Kansas cultivars planted in 2017 are in this list of 26 lines (data not shown), with nine of these 12 lines being sensitive to ToxA. This is significant because the presence of $T s n 1$ applies selection pressure on at least three pathogens to maintain the ToxA gene in the population present in the southcentral hard red winter wheat region of the United States to exploit the $T s n 1$ susceptibility target. Maintaining $T s n 1$ in popular wheat cultivars not only provides a selective advantage to pathogens that currently harbor ToxA but may also provide selection pressure for new or more fit pathogens that acquire ToxA through horizontal transfer.

\section{Literature Cited}

Abeysekara, N. S., Friesen, T. L., Keller, B., and Faris, J. D. 2009 Identification and characterization of a novel host-toxin interaction in the wheat-Stagonospora nodorum pathosystem. Theor. Appl. Genet. 120:117-126.

Ballance, G. M., Lamari, L., Kowatsch, R., and Bernier, C. C. 1996. Cloning, expression and occurrence of the gene encoding the Ptr necrosis toxin from Pyrenophora tritici-repentis. Mol. Plant Pathol. Online. http://www.bspp.org. uk/mppol/1996/1209ballance/.

Chu, C.-G., Friesen, T. L., Xu, S. S., and Faris, J. D. 2008. Identification of novel tan spot resistance loci beyond the known host-selective toxin insensitivity genes in wheat. Theor. Appl. Genet. 117:873-881.

Ciuffetti, L. M., Francl, L. J., Ballance, G. M., Bockus, W. W., Lamari, L., Meinhardt, S. W., and Rasmussen, J. B. 1998. Standardization of toxin nomenclature in the Pyrenophora tritici-repentis/wheat interaction. Can. J. Plant Pathol. 20:421-424.

Ciuffetti, L. M., Tuori, R. P., and Gaventa, J. M. 1997. A single gene encodes a selective toxin causal to the development of tan spot of wheat. Plant Cell 9: 135-144.

Duveiller, E., and Dubin, H. J. 2000. Helminthosporium leaf blights: Spot blotch and tan spot. FAO corporate document repository. http://www.fao.org/docrep/ 006/Y4011E/y4011e0k.htm

Duveiller, E., Kandel, Y. R., Sharma, R. C., and Shrestha, S. M. 2005 Epidemiology of foliar blights (spot blotch and tan spot) of wheat in the plains bordering the Himalayas. Phytopathology 95:248-256.

Faris, J. D., Abeysekara, N. S., McClean, P. E., Xu, S. S., and Friesen, T. L. 2012 Tan spot susceptibility governed by the Tsn1 locus and race-nonspecific resistance quantitative trait loci in a population derived from the wheat lines Salamouni and Katepwa. Mol. Breed. 30:1669-1678.

Faris, J. D., Anderson, J. A., Francl, L. J., and Jordahl, J. G. 1996. Chromosomal location of a gene conditioning insensitivity in wheat to a necrosis-inducing culture filtrate from Pyrenophora tritici-repentis. Phytopathology 86 : 459-463.

Faris, J. D., and Friesen, T. L. 2005. Identification of quantitative trait loci for racenonspecific resistance to tan spot of wheat. Theor. Appl. Genet. 111:386-392.

Faris, J. D., Liu, Z. H., and Xu, S. S. 2013. Genetics of tan spot resistance in wheat. Theor. Appl. Genet. 126:2197-2217.

Faris, J. D., Zhang, Z., Lu, H., Lu, S., Reddy, L., Cloutier, S., Fellers, J. P., Meinhardt, S. W., Rasmussen, J. B., Xu, S. S., Oliver, R. P., Simons, K. J., and Friesen, T. L. 2010. A unique wheat disease resistance-like gene governs effector-triggered susceptibility to necrotrophic pathogens. Proc. Natl. Acad. Sci. U.S.A. 107:13544-13549.

Friesen, T. L., and Faris, J. D. 2012. Characterization of plant-fungal interactions involving necrotrophic effector-producing plant pathogens. Methods Mol. Biol. 835:191-207

Friesen, T. L., Stukenbrock, E. H., Liu, Z. H., Meinhardt, S., Ling, H., Faris, J. D., Rasmussen, J. B., Solomon, P. S., McDonald, B. A., and Oliver, R. P. 2006 Emergence of a new disease as a result of interspecific virulence gene transfer. Nat. Genet. 38:953-956.

Joehanes, R., and Nelson, J. C. 2008. QGene 4.0, an extensible Java QTL-analysis platform. Bioinformatics 24:2788-2789.

Kumar, S., Röder, M. S., Tripathi, S. B., Kumar, S., Chand, R., Joshi, A. K., and Kumar, U. 2015. Mendelization and fine mapping of a bread wheat spot blotch disease resistance QTL. Mol. Breed. 35:218.

Kumar, U., Joshi, A. K., Kumar, S., Chand, R., and Röder, M. S. 2009. Mapping of resistance to spot blotch disease caused by Bipolaris sorokiniana in spring wheat. Theor. Appl. Genet. 118:783-792.

Lamari, L., and Bernier, C. C. 1989. Toxin of Pyrenophora tritici-repentis: Host-specificity, significance in disease, and inheritance of host reaction. Phytopathology 79:740-744.

Liu, Z. H., Friesen, T. L., Ling, H., Meinhardt, S. W., Rasmussen, J. B., and Faris, J. D. 2006. The Tsn1-ToxA interaction in the wheat-Stagonospora nodorum 
pathosystem parallels that of the wheat-tan spot system. Genome 49: $1265-1273$.

Liu, Z. H., Zhong, S., Stasko, A. K., Edwards, M. C., and Friesen, T. L. 2012. Virulence profile and genetic structure of a North Dakota population of Pyrenophora teres f. teres, the causal agent of net form net blotch of barley. Phytopathology 102:539-546.

McDonald, M. C., Ahren, D., Simpendorfer, S., Milgate, A., and Solomon, P. S. 2017. The discovery of the virulence gene ToxA in the wheat and barley pathogen Bipolaris sorokiniana. Mol. Plant Pathol. 19:432-439.

McDonald, M. C., Razavi, M., Friesen, T. L., Brunner, P. C., and McDonald, B. A. 2012. Phylogenetic and population genetic analyses of Phaeosphaeria nodorum and its close relatives indicate cryptic species and an origin in the Fertile Crescent. Fungal Genet. Biol. 49:882-895.

Paillard, S., Schnurbusch, T., Winzeler, M., Messmer, M., Sourdille, P., Abderhalden, O., Keller, B., and Schachermayr, G. 2003. An integrative genetic linkage map of winter wheat (Triticum aestivum L.). Theor. Appl. Genet. 107:1235-1242.
Sievers, F., Wilm, A., Dineen, D., Gibson, T. J., Karplus, K., Li, W., Lopez, R. McWilliam, H., Remmert, M., Söding, J., Thompson, J. D., and Higgins, D. G. 2011. Fast, scalable generation of high-quality protein multiple sequence alignments using Clustal Omega. Mol. Syst. Biol. 7:539.

Tomás, A., and Bockus, W. W. 1987. Cultivar specific toxicity of culture filtrate of Pyrenophora tritici-repentis. Phytopathology 77:1337-1366.

Tuori, R. P., Wolpert, T. J., and Ciuffetti, L. M. 1995. Purification and immunological characterization of toxin components from cultures of Pyrenophora tritici-repentis. Mol. Plant-Microbe Interact. 8:41-48.

Virdi, S. K., Liu, Z., Overlander, M., Zhang, Z., Xu, S. S., Friesen, T. L., and Faris, J. D. 2016. New insights into the roles of host gene-necrotrophic effector interactions in governing susceptibility of durum wheat to tan spot and Septoria nodorum blotch. G3-Genes Genom. Genet. 6:4139-4150.

White, T. J., Bruns, T., Lee, S., and Taylor, J. 1990. Amplification and direct sequencing of fungal ribosomal DNA for phylogenetics. Pages 315-322 in: PCR Protocols: A Guide to the Methods and Applications. M. A. Innis, D. H Gelfand, J. J. Sninsky, and T. J. White, eds. Academic Press, San Diego, CA 